Editorial

\title{
Introduction to a special issue in honour of W. Owen Saxton, David J. Smith and Dirk Van Dyck on the occasion of their 65th birthdays
}

This special issue of Ultramicroscopy, which is dedicated to Owen Saxton, David Smith and Dirk Van Dyck on the occasion of their 65th birthdays, contains original research and review articles written by colleagues who have enjoyed the privilege of working with these three pioneers of quantitative transmission electron microscopy or benefited from their scientific achievements.

A symposium in honour of Dirk Van Dyck was organised by EMAT and held at the University of Antwerp on 21 June 2013. Two colloquia honouring Owen Saxton and David Smith were organised during the PICO 2013 Conference on Frontiers of Aberration Corrected Electron Microscopy, which took place in Kasteel Vaalsbroek from 9 to 12 October 2013 and was organised by the Triebenberg Laboratory in the University of Dresden and the Ernst Ruska-Centre in Forschungszentrum Jülich. The two events attracted more than 200 participants from academia, industry and national laboratories. The content of some of the lectures presented during these events are described in this special issue.

This volume of Ultramicroscopy begins with an overview by Peter Hawkes, which highlights the careers and major scientific achievement of the three guests of honour. The other manuscripts are arranged alphabetically in order of the corresponding author's surname. Each manuscript has been peer-reviewed by two or more referees, to whom the guest editors are grateful for their efficient work.
The pioneering work carried out by Owen Saxton, David Smith and Dirk Van Dyck is recognised in the form of both review articles and original manuscripts, which describe research on quantitative high-resolution transmission electron microscopy, spectroscopy, electron holography, optics and computer simulations. The manuscripts provide a cross-section of the advanced characterisation techniques that can be applied to challenging problems in condensed matter physics, materials science and soft matter research and demonstrate the importance and relevance of Owen Saxton's, David Smith's and Dirk Van Dyck's achievements.

Guest editors

Rafal E. Dunin-Borkowski ${ }^{1}$

Ernst Ruska-Centre, Jülich Research Centre, Germany

Hannes Lichte ${ }^{1}$ Triebenberg Laboratory, Dresden University, Germany

Karsten Tillmann $*, 1$ Ernst Ruska-Centre, Jülich Research Centre, Germany E-mail address: k.tillmann@fz-juelich.de

Sandra Van Aert ${ }^{2}$ EMAT, University of Antwerp, Belgium

Gustaaf Van Tendeloo ${ }^{2}$ EMAT, University of Antwerp, Belgium

\footnotetext{
* Corresponding author. Tel.: +49 2461611438.

1 Organiser of colloquia honouring Owen Saxton and David Smith and Smith and the PICO 2013 Conference on Frontiers of Aberration Corrected Electron Microscopy.

2 Organiser of the colloquium "From Multislice to Big Bang" honouring Dirk Van Dyck.
} 